\title{
A novel in vitro system for gamete fusion in maize
}

\author{
Xiong Bo PENG, Meng Xiang SUN*, Hong Yuan YANG \\ Key Laboratory of Ministry of Education for Plant Developmental Biology, College of Life Sciences, Wuhan University, \\ Wuhan 430072, China
}

\begin{abstract}
Various systems by using electric pulse, calcium, or polyethylene glycol have been developed in the past decade for the in vitro fusion of plant gametes. These in vitro systems provide a new way to study the fertilization mechanisms of plants. In this study, we developed a bovine serum albumin (BSA)-mediated fusion system for the in vitro fusion of maize gametes. The in vitro fusion of the isolated single egg cell and sperm cell of maize was observed microscopically in the BSA solution and the fertilized egg cell showed normal cell wall regeneration and nuclear division. The effects of the BSA concentration, $\mathrm{pH}$ value and calcium level on the efficiency of the maize gamete fusion were also assessed. BSA concentration and $\mathrm{pH}$ value did significantly affect the efficiency of the gamete fusion. Calcium was not necessary for the gamete fusion when BSA was present. The optimal solution for the gamete fusion contained $0.1 \% \mathrm{BSA}, \mathrm{pH} 6.0$. The fusion frequency was as high as $96.7 \%$ in that optimal solution. This new in vitro fertilization system offers an alternative tool for the in vitro study of fertilization mechanisms with much simpler manipulating procedure than PEG system, and it will be especially useful for the in vitro study of the calcium dynamics during plant fertilization.
\end{abstract}

Keywords: in vitro fertilization, bovine serum albumin, calcium, maize.

\section{INTRODUCTION}

Fertilization in higher plants occurs deeply in the maternal tissue and is therefore inaccessible for experimental manipulation [1]. The establishment of in vitro fertilization (IVF) techniques would greatly facilitate the investigation of the molecular and cellular events of fertilization. In the past decade, various in vitro fertilization techniques have been developed for higher plants. The fusion of isolated gametes was induced by using electric pulse [2], polyethylene glycol [3-5], or calcium [6-8]. With the help of the established IVF systems great progress has been made for some key events concerning fertilization mechanisms such as polygamy, gamete recognition and nuclear behavior in fused gametes [4, 9-11], which were actually unable to be investigated by traditional methods. However, IVF is still a challenge work and skill-dependent technique to researchers. Therefore it is still needed to further improve the technique of IVF, especially the technique of

*Correspondence: Meng Xiang SUN

E-mail:mxsun@whu.edu.cn

Abbreviations: BSA (bovine serum albumin); IVF (in vitro fertilization); PEG (polyethylene glycol). gamete protection and gamete fusion, for further simplifying the procedure and making it easier to manipulators.

In this study, we investigated the possibility of the in vitro plant gamete fusion using BSA medium. BSA was previously used in medium as a protectant for the storage of isolated sperm cells in our experiments, and sperm cells was much more protected and was viable longer in BSA medium than in other medium [12]. We supposed that the gamete fusion in BSA medium may be possible if the concentration of BSA is adjusted to an appropriate level. The objective of this study was to develop a simple and efficient BSA-induced fusion system for the in vitro fertilization of maize gametes.

\section{MATERIALS AND METHODS \\ Plant material}

Maize (Zea mays L.) plants of inbred lines A 188 were grown in a greenhouse under $16 \mathrm{~h}$ photoperiod at $25^{\circ} \mathrm{C}$. The ears of the plants were bagged before the silk emergence to prevent pollination. Ears were collected at a receptive stage when emerged silks reached 12-13 $\mathrm{cm}$ in length [13].

\section{Isolation of egg cells and sperm cells}

Egg cells were isolated from ovules dissected from mature ears of maize using a method modified from Kranz et al [2]. Half-ovules were 
incubated for $5 \mathrm{~min}$ at room temperature in an enzymatic solution containing 0.5\% Macerozyme Onozuka R10 (Yakult Honsha, Tokyo, Japan), 0.5\% Cellulase Onozuka R10 (Yakult Honsha, Tokyo, Japan), adjusted to $725 \mathrm{mosmol} / \mathrm{kg} \mathrm{H}_{2} \mathrm{O}$ with mannitol, $\mathrm{pH}$ 5.7. Ovule pieces were then washed with $12 \%$ mannitol and egg cells were gently picked out from the embryo sac by manual microdissection under an inverted microscope (Olympus CK-30).

Sperm cells were released from freshly collected pollen grains after an osmotic shock in $12 \%$ mannitol, and isolated followed the method described by Kranz et al [2].

\section{Gamete Fusion}

The fusion of one egg cell and one sperm cell was performed in a $3.5 \mathrm{~cm}$ diameter plastic Petridishe that was previously filled with 2 ml BSA (Roche) fusion medium. Different BSA concentrations (0-0. $6 \%), \mathrm{pH}$ values (5-8) and calcium levels ( $0-2 \mathrm{mM}$ or adding $2 \mathrm{mM}$ EGTA) were assessed for their effects on the adhesion and fusion of the gametes. Based on the results of these tests, the standard optimal fusion medium used in this study contained $3 \mathrm{mM} 2[\mathrm{~N}$-morpholino] ethanesulfonic acid (MES), $0.1 \%(\mathrm{~W} / \mathrm{V}) \mathrm{BSA}$, and adjusted to 750 mosmol $/ \mathrm{kg} \mathrm{H}_{2} \mathrm{O}$ with mannitol, $\mathrm{pH}$ 6.0. As a control, the fusion of male and female gametes was also performed in PEG medium containing $5 \mathrm{mM}$ calcium, $3 \mathrm{mM}$ MES, 20\% (W/V) PEG and 5\%(W/V) mannitol, pH 6.0.

The fusion process was observed under an inverted microscope (Olympus CK-30). The individual pairs of male and female gametes were put into contact by a microcapillary. At least 20 pairs of cells were used for the fusion in each experiment.

\section{Culture of the fusion products}

Fertilized egg cells were cultured in droplets of the modified basic MS medium [14]. The medium were adjusted to $780 \mathrm{mOsmol} / \mathrm{kg}$ $\mathrm{H}_{2} \mathrm{O}$ with $4 \%$ glucose and $11 \%$ mannitol, $\mathrm{pH}$ 5.7. Each of the droplets contained 2-4 $\mu 1$ of medium and covered by mineral oil in a 3.5 $\mathrm{cm}$ diameter glass Petri dish. The culture dishes were kept in a culture chamber in dark at $25^{\circ} \mathrm{C}$ to encourage the further development of fusion products.

\section{Cell wall staining}

For staining cell wall, zygotes were incubated in $0.001 \%$ Fluorescent Brightener 28 (Sigma) solution for $10 \mathrm{~min}$. The Fluorescent Brightener 28 was dissolved in $12 \%$ mannitol. Then, the zygotes were washed three times in $12 \%$ mannitol before observation.

\section{Microscopy and image processing}

For visualizing the process of the gamete fusion, cells were examined under an inverted microscope (Olympus IMT-2), equipped with a cooled charge-coupled device (CCD) camera (CoolSNAPHQ, Roper Scientific Inc.). Digital images from CCD were captured on a computer equipped with the software of Image-Pro Express (Media Cybernetics Inc.). Using the sequential image grabbing function, an image was acquired at a maximum rate of $0.4 \mathrm{~s}$ every frame.

Both Cultured cells and the cells after staining with Fluorescent Brightener 28 were observed under an epifluorescence inverted microscope (Leica DM IRE2), equipped with a cooled CCD (RTE/ CCD-1300-Y/HS, Roper Scientific Inc.).

Digital images from CCD were captured on a computer equipped with the software of MetaMorph (Universal Imaging Corporation Inc.). Images were processed using Photoshop 6.0 software (Adobe
Systems, Mountain View, CA).

\section{Online supplementary material}

Video 1(http://www.cell-research.com/20059/video/video1.mpg) shows the fusion process of the isolated sperm cell and egg cell of maize in the BSA fusion medium.

\section{RESULTS}

\section{The development of BSA-mediated fusion system}

No adhesion and fusion was observed when gametes were contacted in the fusion medium without BSA. When the concentration of BSA was $0.01 \%$, the adhesion of gametes was accomplished in 10-30 min and in this case the fusion frequency was $15.0 \%$. When the concentration of BSA was either $0.1 \%$ or $0.6 \%$, the gametes adhere to each other in $10 \mathrm{~s}$, and their fusion frequency was increased significantly up to $96.7 \%$ or $95.0 \%$ respectively (Fig. 1).

The role of BSA in gamete fusion was further investigated by altering the $\mathrm{pH}$ value of fusion medium. When the $\mathrm{pH}$ value was 8.0 , no gamete adhesion was observed and consequently no fusion occurred. When the $\mathrm{pH}$ value was 7.0 , the fusion frequency was $24.0 \%$. When the $\mathrm{pH}$ value decreased further to 6.0 , the fusion frequency was increased sharply to $96.7 \%$. After $2 \mathrm{~min}$ in $0.1 \%$ BSA of pH 5.0 or lower, sperm cells and egg cells broke and lost their integrity. Sperm cells could be maintained the ability to fusion with egg cells more than $60 \mathrm{~min}$ in $0.1 \% \mathrm{BSA}$ at pH 6.0 to 8.0 (Fig. 2).

Without adding calcium in the medium, $96.7 \%$ gametes could easily fuse in $0.1 \% \mathrm{BSA}$ of $\mathrm{pH} 6.0$. Adding $2 \mathrm{mM}$ EGTA in the medium resulted in $60.0 \%$ gametes fusion. In contrast, adding $2 \mathrm{mM}$ calcium made gamete adhesion more difficult and no fusion occurred. Adding $0.5 \mathrm{mM}$ calcium resulted in the fusion of just $30.0 \%$ cell pairs. Whereas,

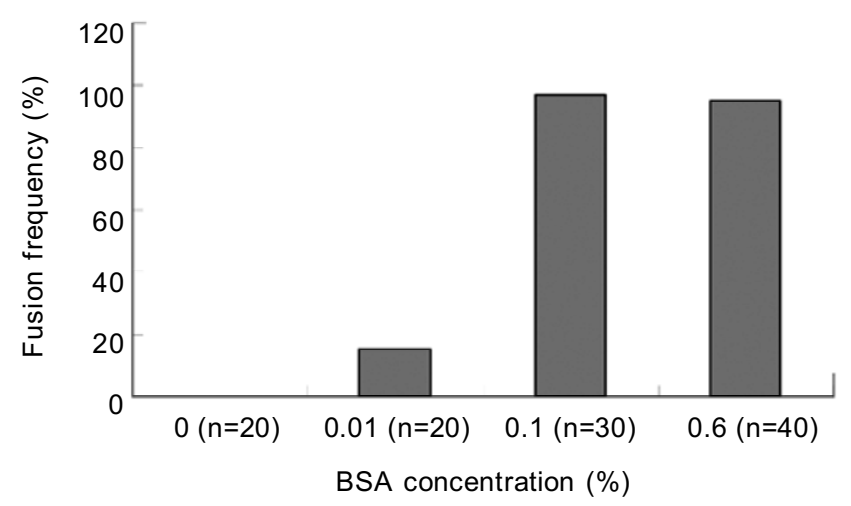

Fig. 1 Effects of different concentrations of bovine serum albumin (BSA) on the fusion frequency of the isolated sperm cell and egg cell of maize. The fusions were carried out in fusion medium of $\mathrm{pH} 6.0$ without external calcium. The values of $\mathrm{n}$ represent the total number for fusion in each independent experiment. 


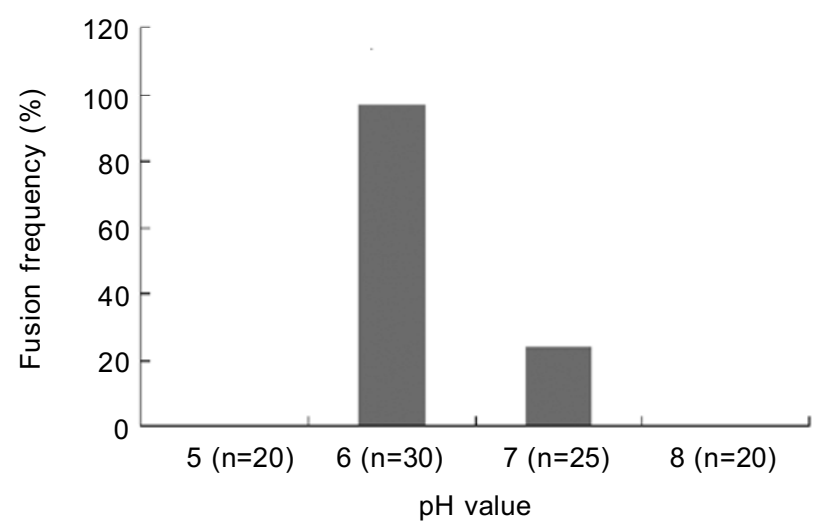

Fig. 2 Effects of different $\mathrm{pH}$ value on the fusion frequency of isolated sperm cell and egg cell of maize. The fusions were carried out in $0.1 \%$ BSA without external calcium. The values of $\mathrm{n}$ represent the total number for fusion in each independent experiment.

adding $0.1 \mathrm{mM}$ calcium the fusion frequency could be up to $95 \%$ (Fig. 3).

\section{Cytological characteristics of the in vitro fusion}

Under the optimal BSA medium, the gamete fusions were usually occurred 7-10 min after adhesion. Two fusion processes were observed: the quick process and the slow process. During the quick fusion process, the sperm cell suddenly entered into the egg and the whole process completed within 1-2 s. In this process, the digital images of the process could not easily be captured. During the slow process, the sperm cell quickly pressed to the egg cell in the first $2 \mathrm{~s}$ (Fig. 4B-D). The sperm cell was tightly attached with the egg cell in the following $6 \mathrm{~s}$ (Fig. 4E and $4 \mathrm{~F}$ ) and seemed there was an interaction between the membranes of two gametes in this period. Then the sperm moved again to press the egg cell in successive $10 \mathrm{~s}$ (Fig. $4 \mathrm{G}$ and $4 \mathrm{H})$ and the whole sperm cell finally entered the egg cell (Fig. 4I). The whole fusion process was finished

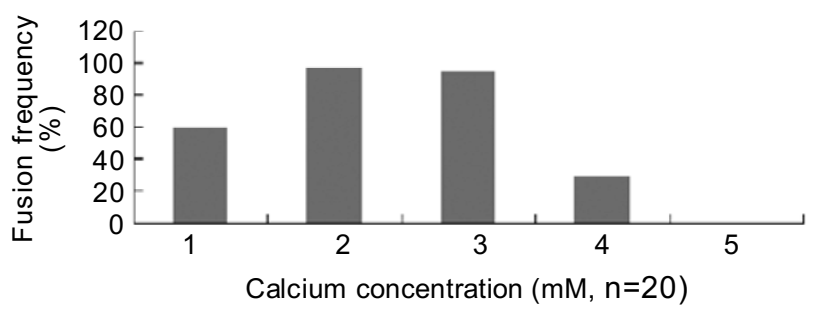

Fig. 3 Effects of calcium concentrations on the fusion frequency of isolated sperm cells and egg cells of maize. The fusions were carried out respectively. 1) $0.1 \%$ BSA with 2 mM EGTA; 2) $0.1 \%$ BSA; 3) $0.1 \%$ BSA with $0.1 \mathrm{mM}$ calcium; 4) $0.1 \%$ BSA with $0.5 \mathrm{mM}$ calcium; 5) $0.1 \%$ BSA with $2 \mathrm{mM}$ calcium. The values of $\mathrm{n}$ represent the total number for fusion in each independent experiment.

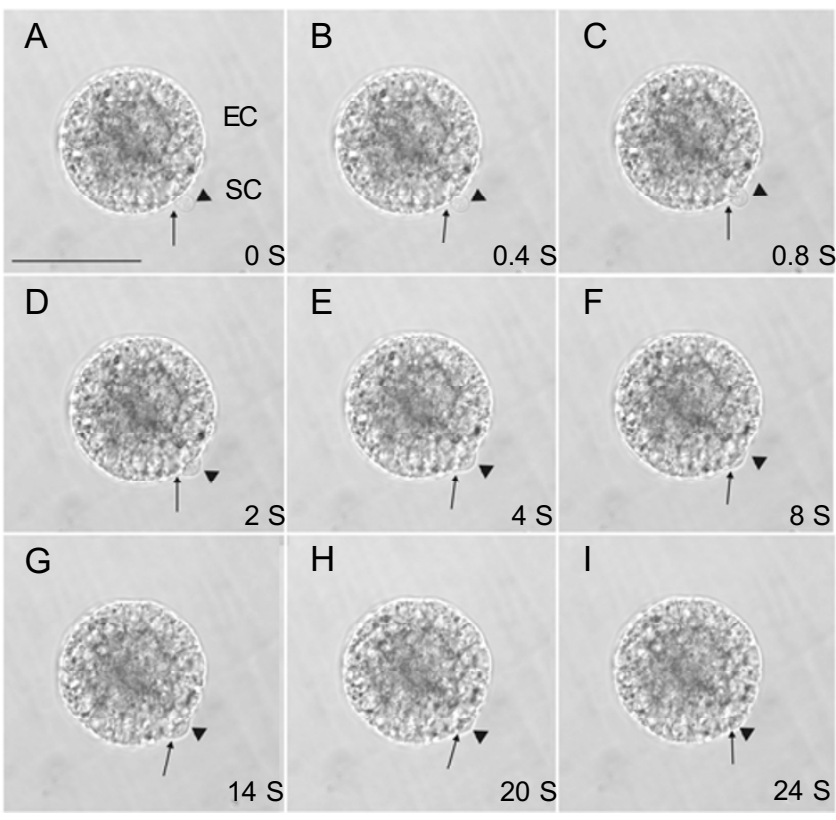

Fig. 4 Timelapse images of in vitro adhesion (A) and fusion (B-I) of the maize sperm cell (SC) and egg cell (EC) induced by $0.1 \%$ BSA medium ( $\mathrm{pH}$ 6.0). Images were taken at varying time intervals over a period of $24 \mathrm{~s}$, as indicated in right-bottom corners of each image. The arrows indicate fusion site and arrowheads indicate sperm cell. Bar $=50 \mu \mathrm{m}$.

in $24 \mathrm{~s}$ and was visualized (Fig. 4 and video 1).

Before fertilization the isolated egg cell was roundshaped (Fig. 5A) and its nucleus contained one nucleolus (Fig. 5B). Within several minutes after the fusion, the egg cell underwent a transient and reversible contraction (data not shown) as described by previous studies [15]. After incubation for $20 \mathrm{~h}$ in MS medium, some zygotes showed a little shrinkage and the cell wall could be clearly observed (Fig. 5C and 5D). After $24 \mathrm{~h}$ incubation in MS medium, fusion products showed two nucleoli in their nucleus (Fig. 5E), which is a marker showing nuclear fusion already completed. After $40 \mathrm{~h}$ incubation in MS medium, the nuclear division of zygotes was observed (Fig. $5 F)$. These results indicated that the artificial zygotes produced by our IVF system were well developed.

In PEG control medium, $100 \%$ fusion frequency was observed among 20 pairs of gametes but the gamete fusion behavior was different from that in BSA fusion medium. Once the pair of gametes adhered, the sperm cell pressed to egg cell and whole sperm cell entrapped into egg cell in $10 \mathrm{~s}$ (Fig. 6) and it took another 3-10 min for the sperm cell to enter into the egg cell.

\section{DISCUSSION}

In this investigation, a novel in vitro system for maize 


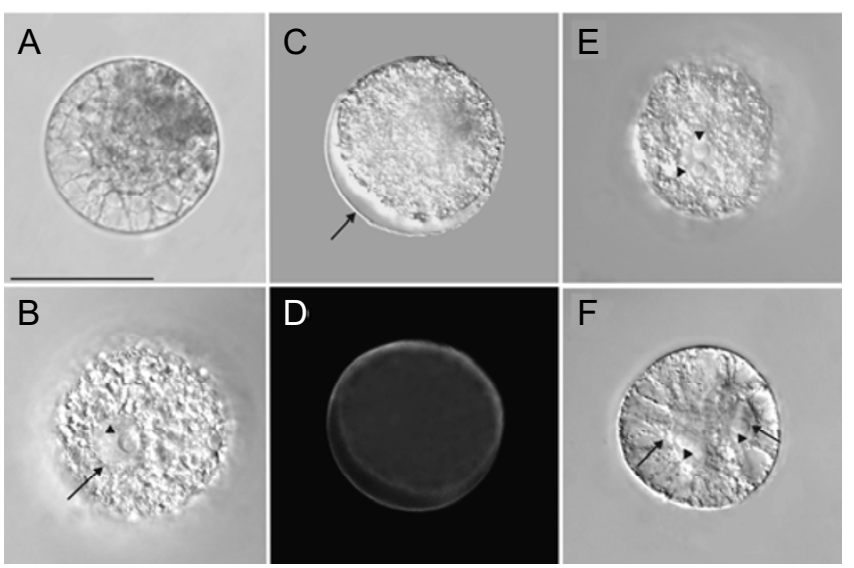

Fig. 5 The development of in vitro fertilized egg cell of maize. (A) A round-shaped egg cell. (B) The egg showing nucleus (arrow) and nucleolus (arrow head). (C) A shrank zygote $18 \mathrm{~h}$ after culture. The arrow indicates well regenerated cell wall of the zygote. (D) Fluorescent image showing cell wall of the same zygote as in $\mathrm{C}$. The cell wall was labeled by Fluorescent Brightener 28. (E) A zygote $20 \mathrm{~h}$ after fusion, showing two nucleoli (arrow heads) already appeared in the nucleus. Note the diameters of the two nucleoli are different. (F) A zygote $40 \mathrm{~h}$ after fusion, showing nuclear division. The arrow heads indicate two nucleoli and arrows indicate two nuclei respectively. Bar $=50 \mu \mathrm{m}$.

gametes fusion was developed. The fusion frequency was as high as $96.7 \%$ in the solution containing $0.1 \%$ BSA at $\mathrm{pH}$ 6.0. The whole fusion process was observed and the zygotes were well developed.

BSA has been widely used in the media for the capacitation of spermatozoa and the acrosome reaction of mammalian sperm [16]. It is well known that mammalian sperm undergoes biochemical and physiological changes in the female reproductive tract, termed capacitation, which enables sperm fertilization [17]. However, it was not clear whether angiosperm sperm cells also undergo capacitation for fertilization. In this investigation, we showed, to our knowledge, in the first time that BSA did have the capacity to induce gametes adhesion and fusion in vitro. Previous study indicated that BSA conformation could change in different $\mathrm{pH}$ value [18]. Our result showed that the frequency of gamete adhesion and fusion decreased with the decrease of $\mathrm{pH}$ value (Fig. 5). The effect of $\mathrm{pH}$ value on the fusion frequency may result from its effects on BSA conformation and therefore influence its function in mediating cell fusion.

Generally, BSA induced gamete fusion shows several advantages over PEG induced fusion. In BSA medium both isolated gametes and fusion products showed vigorous viability demonstrated by FDA test and their fusion behavior. In fact, BSA was previously used as a protectant and could obviously prolong the storage of generative cells and sperm cells of higher plants [12]. The isolated gametes could be kept in BSA for hours before and after fusion. However in PEG medium the gametes should be fused as soon as possible to avoid harmful effect of PEG on the membrane of the fusion products. BSA fusion system is also simpler than PEG system. It takes 3-10 min to bring the gametes into contact in PEG medium for its viscosity. In contrast, the cells can settle down quickly at the bottom of Petri dish in BSA medium and it is easy to move them close to each other afterwards. It takes less than $10 \mathrm{~s}$ to bring the male and female gametes into contact for the fusion.

As described in our previous work [4], adding low concentration of calcium in PEG can obviously enhance gamete fusion, however, it also makes cells easy to stick on the bottom of Petri dish and thus they are no longer to be moved for fusion. In this case, it needs to disturb the cells all the time to keep them being suspended in the medium. In BSA medium, however, the cells can be moved freely in the medium and once the pairs of gametes are adhered in BSA medium, they can be left there more than $10 \mathrm{~min}$ for observation of their fusing process without worrying their sticking on glass bottom. This is especially useful for less experienced researchers to manipulate.

It has been shown that calcium plays a pivotal role in
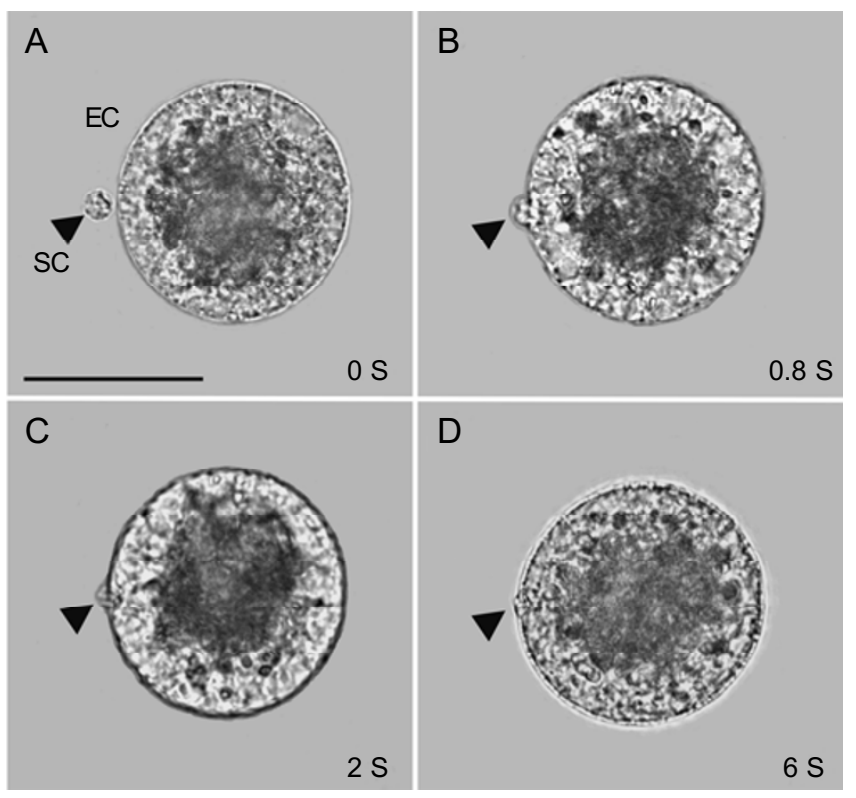

Fig. 6 Timelapse images of in vitro adhesion process (A-D) of maize sperm cell (SC) and egg cell (EC) induced by PEG medium containing $5 \mathrm{mM}$ calcium, $3 \mathrm{mM}$ MES, 20\% (W/V) PEG and 5\% $(\mathrm{W} / \mathrm{V})$ mannitol, $\mathrm{pH}$ 6.0. Images were taken at varying time intervals over a period of $6 \mathrm{~s}$, as indicated in right-bottom corners of each image. The arrowheads indicate sperm cell. $\mathrm{Bar}=50 \mu \mathrm{m}$. 
membrane fusion in many plant cell systems [19]. For IVF, calcium had been used at high concentrations (50 $\mathrm{mM}$ ) to induce gamete fusion of maize [7]. Faure et al [6] showed that the fusion efficiency of IVF of maize in low calcium $(1 \mathrm{mM})$ was much lower than that in high calcium $(5 \mathrm{mM})$. Although fusion could happen at even lower calcium concentrations (around $100 \mu \mathrm{M}$ ), the fusion frequency was much lower [20]. It seemed that calcium at millimolar levels would benefit IVF. In deed, our study also showed that the PEG medium containing calcium was much better than that of without calcium to induce fusion of maize gametes. Adding calcium in fusion medium obviously shortened the duration of gamete adhesion and fusion (data not shown). However, in our present experiments, $97.6 \%$ gametes could easily fuse without adding calcium and $60 \%$ gametes could easily fuse with the $2 \mathrm{mM}$ EGTA in the medium. The results indicated that adding calcium was not necessary for gamete fusion with the presence of BSA in the medium, although the low concentration of calcium at micromorlar level might benefit gametes for their adhesion and fusion.

It was reported that maize gamete fusion could trigger a calcium influx, which was then followed by an increase of cytoplasmic calcium [21]. In this process, the extracellular calcium that penetrated the egg cells may partly contribute to the cytosolic calcium increase. In BSA induced gamete fusion, as demonstrated in present work, gamete fusion could also occur in presence of $2 \mathrm{mM}$ EGTA. This new fusion system therefore offers an alternative way and useful control for investigating the link of the calcium influx to the cytosolic calcium increase during IVF.

\section{ACKNOWLEDGEMENTS}

We would like to thank Prof. E. Kranz (University of Hamburg, Germany) for providing us Maize (Zea mays L.) seeds of inbred lines A 188. This research was supported by the National Natural Science Foundation of China (30370743, 90408002), National Science Found for Distinguished Young Scholars of China (30225006) and Supported by Program for Changjiang Scholars and Innovative Research Team in University (PCSIRT).

Received, May 6, 2005

Revised, Aug 29, 2005

Accepted, Sep 13,2005

\section{REFERENCES}

1 Russell SD. Double fertilization. Int Rev Cytol 1992; 140:357-88.

2 Kranz E, Bautor J, Lörz H. In vitro fertilization of single, isolated gametes of maize mediated by electrofusion. Sex Plant Reprod 1991; 4:12-6.

3 Sun MX, Yang HY, Zhou C. Single-pair fusion of various combi- nations between female gametoplasts and other protoplasts in Nicotiana tabacum. Acta Bot Sin 1995; 36:489-93.

4 Sun MX, Moscatelli A, Yang HY, Cresti M. In vitro double fertilization in Nicotiana tabacum (L.): fusion behavior and gamete interaction traced by video-enhanced microscopy. Sex Plant Reprod 2000; 12:267-75.

5 Tian HQ, Russell SD. Micromanipulation of male and female gametes of Nicotiana tabacum. II. Preliminary attempts for in vitro fertilization and egg cell culture. Plant Cell Rep 1997; 16: 657-61.

6 Faure JE, Digonnet C, Dumas C. An in vitro system for adhesion and fusion of maize gametes. Science 1994; 263:1598-600.

7 Kranz E, Lörz H. In vitro fertilisation of maize by single egg and sperm cell protoplast fusion mediated by high calcium and high pH. Zygote 1994; 2:125-8.

8 Kranz E, Kumlehn J. Angiosperm fertilisation, embryo and endosperm development in vitro. Plant Sci 1999; 142:183-97.

9 Faure JE, Rusche ML, Thomas A, et al. Double fertilization in maize: the two male gametes from a pollen grain have the ability to fuse with egg cells. Plant J 2003; 33:1051-62.

10 Lord EM, Russell SD. The mechanisms of pollination and fertilization in plants. Annu Rev Cell Dev Biol 2002; 18:81-105.

11 Sun MX, Moscatelli A, Yang HY, Cresti M. In vitro double fertilization in Nicotiana tabacum (L.): polygamy compared with selected single pair somatic protoplast and chloroplast fusions. Sex Plant Reprod 2000; 13:267-75.

12 Mo YS, Yang HY. Mass isolation and preservation of viable sperm cells in Brassica campestris var. purpurea. Acta Bot Sin 1991; 33:649-57.

13 Dupuis I, Dumas C. In vitro pollination as a model for studying fertilization in maize (Zea mays L.). Sex Plant Reprod 1989; 2: 265-9.

14 Murashige T, Skoog, F. A revised medium for rapid growth and bioassays with tobacco tissue cultures. Plant Physiol 1962; 15: 473-97.

15 Kranz E, Wiegen PV, Lörz H. Early cytological events after induction of cell division in egg cells and zygote development following in vitro fertilization with angiosperm gametes. Plant $\mathrm{J}$ 1995; 8:9-23.

16 Bavister BD, Yanagimachi R. The effects of sperm extracts and energy sources on the motility and acrosome reaction of hamster spermatozoa in vitro. Biol Reprod 1977; 16:228-37.

17 Chang MC. Fertilizing capacity of spermatozoa deposited into fallopian tubes. Nature 1951; 168:697-8.

18 Foster JF. Some Aspects of the Structure and Conformational Properties of Serum Albumin. In: Rosenoer VM, Oratz M, Rothschild MA, eds. Albumin Structure, Function and Uses. Pergamon. Pergamon Press: Oxford 1977:53-84.

19 Boss WF. Fusion-permissive protoplasts: a plant system for studying cell fusion. In: Sowers AE, ed. Cell fusion. Plenum Press: New York 1987:145-66.

20 Antoine AF, Faure JE, Cordeiro S, et al. A calcium influx is triggered and propagates in the zygote as a wavefront during in vitro fertilization of flowering plants. Proc Natl Acad Sci U S A 2000; 97:10643-8.

21 Antoine AF, Faure JE, Dumas C, Feijo JA. Differential contribution of cytoplasmic $\mathrm{Ca}^{2+}$ and $\mathrm{Ca}^{2+}$ influx to gamete fusion and egg activation in maize. Nat Cell Biol 2001; 3:1120-3.

Edited by Lu LIANG 